\title{
Understanding User Experience in Stereoscopic 3D Games
}

\author{
Jonas Schild \\ Entertainment Computing Group \\ University of Duisburg-Essen \\ Forsthausweg 2, \\ 47057 Duisburg, Germany \\ jonas.schild@uni-due.de
}

\author{
Joseph J. LaViola Jr. \\ University of Central Florida \\ Department of EECS \\ 4000 Central Florida Blvd. \\ Orlando, FL 32816 USA \\ jj1@eecs.ucf.edu
}

\author{
Maic Masuch \\ Entertainment Computing Group \\ University of Duisburg-Essen \\ Forsthausweg 2, \\ 47057 Duisburg, Germany \\ maic.masuch@uni-due.de
}

\begin{abstract}
Recent advances in digital game technology are making stereoscopic games more popular. Stereoscopic 3D graphics promise a better gaming experience but this potential has not yet been proven empirically. In this paper, we present a comprehensive study that evaluates player experience of three stereoscopic games in comparison with their monoscopic counterparts. We examined 60 participants, each playing one of the three games, using three selfreporting questionnaires and one psychophysiological instrument. Our main results are (1) stereoscopy in games increased experienced immersion, spatial presence, and simulator sickness; (2) the effects strongly differed across the three games and for both genders, indicating more affect on male users and with games involving depth animations; (3) results related to attention and cognitive involvement indicate more direct and less thoughtful interactions with stereoscopic games, pointing towards a more natural experience through stereoscopy.
\end{abstract}

\section{Author Keywords}

Games; stereoscopy; player experience; game experience; $\mathrm{S} 3 \mathrm{D}$; play; genre; presence; immersion; simulator sickness.

\section{ACM Classification Keywords}

H.5.m [Information Interfaces and Presentation (e.g., HCI)]: Miscellaneous; K.8.0 [Personal Computing]: General Games.

\section{General Terms}

Experimentation; Human Factors.

\section{INTRODUCTION}

Since the success of James Cameron's Avatar, many new digital media productions have promoted stereoscopic 3D vision as a strong benefit, be it movies, 3DTV, or games. The Sony Playstation 3 and the Nintendo 3DS offer stereoscopic 3D (S3D) gaming on consoles while driverbased solutions such as Nvidia 3D Vision or TriDef let the

Permission to make digital or hard copies of all or part of this work for personal or classroom use is granted without fee provided that copies are not made or distributed for profit or commercial advantage and that copies bear this notice and the full citation on the first page. To copy otherwise, or republish, to post on servers or to redistribute to lists, requires prior specific permission and/or a fee.

CHI'12, May 5-10, 2012, Austin, Texas, USA.

Copyright 2012 ACM 978-1-4503-1015-4/12/05...\$10.00.
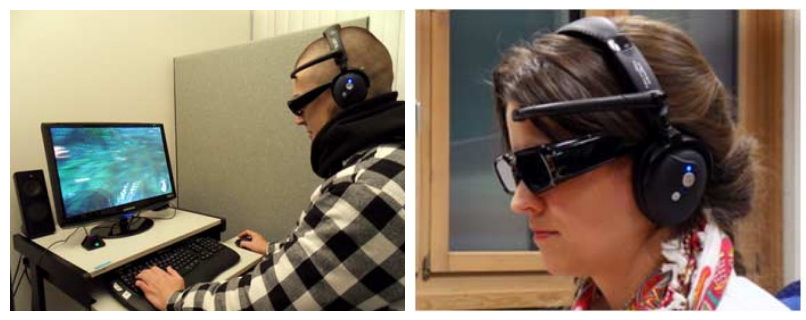

Figure 1: Setup (left) and equipment (right) consisting of Nvidia 3D Vision glasses, MindSet NeuroSky, and ear plugs beneath the headset.

user play in $\mathrm{S} 3 \mathrm{D}$ on the $\mathrm{PC}$. In both cases, existing games and well known game concepts are extended with S3D vision as a toggle option. Nevertheless, to have such an option, players are required to purchase new display systems, glasses, or consoles. It remains unclear whether S3D actually offers a substantial additional value to players. Industry representatives announce S3D gaming as "the most exciting and engaging way to deliver an immersive game experience" ${ }^{1}$. While recent studies actually indicate an increased engagement and improved experience, the reason for such an effect is not understood (e.g., game performance is not necessarily improved by $\mathrm{S} 3 \mathrm{D}$ vision [16,24]). In addition, negative impacts on user experience through S3D are known as well: visual discomfort is expected to occur, especially at intensive depth levels involving large parallax settings or drastic changes in depth [9].

We expect subjective player experience in $\mathrm{S} 3 \mathrm{D}$ gaming to differ both positively and negatively from monoscopic gameplay. In this paper, we focus on understanding the quality of the actual impact and why users feel the way they do when playing games in 3D stereo over a 2D display. How do different game concepts and target groups affect the experience? Can we automatically improve every experience with S3D, as often promised by industry and expected by users? By answering these questions, game designers can begin to take this knowledge and use it to assist in developing more compelling 3D stereo games.

\footnotetext{
${ }^{1}$ see http://www.thegamecreators.com/pages/newsletters/ne wsletter_issue_86.html\#10
} 
We conducted a study among 60 participants to compare subjective player experience of three different games played in S3D and in monoscopic vision. The study applies four empirical tools: questionnaires on game experience, spatial presence and simulator sickness, and the NeuroSky MindSet.

\section{RELATED WORK}

\section{Benefits and Disadvantages of Stereoscopic Vision}

The impact on experience of playing games using S3D compared to regular monoscopic displays is unknown. Such a subjective experience while playing a game is often referred to as user experience [1], player experience [20], or game experience. Positive and negative effects of stereoscopic vision on user experience have been intensively studied in the domain of image perception, $3 \mathrm{DTV}$, and with virtual reality applications. According to Tam et al., S3D image sequences are generally preferred over non-stereoscopic versions, perceived depth is rated greater for S3D sequences than for non-stereoscopic ones, and perceived sharpness of stereoscopic sequences is rated the same or lower compared to non-stereoscopic sequences [30]. In addition, animations in depth are perceived faster in S3D [23]. S3D also has a powerful impact on providing information about spatial location, size, shape, or orientation of 3D objects [10]. Virtual scenes [13] and video clips [11] experienced in S3D induce an increased perceived presence. The latter study also reports more attention towards details requiring more time to explore a S3D scene. S3D images are generally perceived more naturally and induce a better viewing experience [27].

In contrast to these benefits, current stereoscopic displays with a fixed screen plane cause several human visual cues to conflict with each other; the most prominent ones being accommodation and convergence. This conflict is known to result in a negative experience due to visual discomfort [17] associated with symptoms of visual fatigue (e.g., sore eyes, eye strain, headache, blurred vision) [7]. A common solution is to reduce parallax settings to a comfortable viewing range [28], effectively limiting stereoscopic effects. Additional distortions occurring from exaggerated disparity may include cross-talk, binocular rivalry, blur, compression artifacts, noise [34], or geometric distortions [33]. For objective assessment of image quality, 2D image quality metrics are not appropriate and 3D quality metrics are not available yet [34]. In addition, the perceived quality of stereoscopic presentations depends on the 3D display (driver, electronics), varying with individual user physiology [8]. Even watching good quality 3DTV induced symptoms of simulator sickness in a pre-/post-comparison [22]. Such symptoms are already noticeable after less than five minutes of exposure [14].

\section{Stereoscopy in Digital Games}

There has been little academic research on player experience with stereoscopic gaming. Zachara and Zagal reviewed the failure of Nintendo's Virtual Boy. They describe a lack of focused design and emphasize a need for stereoscopic game mechanics [35]. Rajae-Joordens evaluated gameplay of Quake III: Arena on an autostereoscopic, lenticular display for 2D vs. S3D display modes [24]. S3D caused an increase in emotional arousal while the heart rate remained unaffected. A questionnaire showed significantly higher presence and engagement results for S3D. Additionally, no visual symptoms or simulator sickness was found. The gaming performance (score) initially was higher in S3D but equal to 2D after prolonged use. Most of the participants (85\%) preferred S3D over 2D. LaViola and Litwiller also investigated user performance benefits of S3D with 40 participants playing five current digital games. Their results indicate that although participants preferred playing in S3D for the tested games, it did not provide any significant advantage in overall user performance and learning. Still, the users reported an improved experience and increased engagement while playing in S3D. Interestingly, the variety of the evaluated games showed no influence to these results [16].

In a recent study, Takatalo et al. evaluated user experience of Need for Speed Underground (a racing game published in 2003) played at a resolution of $800 \times 600$ pixels in S3D with two different parallax settings and monoscopic vision using a multifactorial between-subjects design [29]. Compared to monoscopic vision, they found a higher presence experienced with medium parallax settings only, not with high settings, probably due to visual discomfort. With their slightly lower resolution setting, they did not find any effect in emotional factors such as the experienced flow, fun or enjoyment. However, in another study, novel display technology (e.g., higher resolution) was found to amplify impact from S3D on user experience [32].

Overall, previous work proposes that $\mathrm{S} 3 \mathrm{D}$ in some games is generally preferred. However, it remains unclear exactly how player experience actually benefits from stereoscopic presentation. How does it relate to known advantages of stereoscopy in general? What is the impact of varying game design, modern stereoscopic display technology and rendering quality on novel stereoscopic gaming experience?

\section{USER STUDY}

The aim of our study was to examine how player experience differs between playing a game being presented in stereoscopic vision (SV) and playing it in monoscopic vision (MV). We hypothesize that:

1. Stereoscopy results in a different game experience compared to a monoscopic display.

2. Playing stereoscopic games confirms benefits and flaws of experiencing other stereoscopic visuals (e.g., movies, 3DTV), resulting in increased presence and increased simulator sickness.

3. These results are independent of the game.

The participants were tasked with playing through preselected sections of one of three different games. 


\section{Selecting the Games}

Before the study, more than 20 S3D-enabled games were tested based on several suitability criteria. None of the games were primarily designed with stereoscopy in mind but provided stereoscopic vision as an option, provided through the $3 \mathrm{D}$ vision driver. Other $3 \mathrm{D}$ related aspects (ghosting, double vision) were tested subjectively on the test system (glasses, display, computer, display driver) as recommended before. Besides stereoscopic properties, we focused on game playability. The games needed to provide easy entry to keep the whole test setting within a reasonable time-frame and suitable to untrained players who might have never held a gamepad before. Game controls thus had to be similar between the games and should avoid a possible impact from active body movements on psychophysiological measures (i.e., on a steering wheel compared to body passiveness when a game only requires single keystrokes). Hence, all selected games had to be playable using cursor keys and a few additional keys on the keyboard. The games needed to appeal to a broad audience but also needed to differ significantly in genre and visual setup. The latter aspect refers to the strong depth effect of stereoscopy on the user. For each game, two parts were selected, one for each experimental session. The parts were selected to offer similar game mechanics and tasks within each game while allowing gameplay for both casual and experienced players. The S3D settings for each game were set to provide a comfortable viewing on the used display.

James Cameron's Avatar: The Game, UbiSoft, 2009 (AV) $\mathrm{AV}$ is a $3 \mathrm{D}$ action game played in third person view. The player controls the player character through a 3D game world and has full control over the camera view in a certain range around the player model. The task is to explore the 3D world, to collect items, to interact with non-player characters, and to shoot enemy creatures. The first session consisted of the first level of the game, the second session started with the middle part of the second level.

Blur, ActivisionBlizzard, 2010 (BL)

$\mathrm{BL}$ is a racing game where the player controls a car from a third-person view while the camera moves into depth. This effect is exaggerated when the player switches into a boost mode, drastically increasing the speed. Six races at medium difficulty were selected, three from the first and three from the second level.

\section{Trine, Nobilis/Frozenbyte, 2009 (TR)}

$\mathrm{TR}$ is an action-adventure game where the player controls one of three different fantasy characters through a sidescrolling world with primarily lateral camera animations. The world is constructed from 3D models and spans across several depth planes, thus leaving a spatial impression. For the playing sessions we selected the start of the game and the first part of the second level.

\section{Participants}

We recruited 60 participants (26 male, 34 female) from our university, all students. The study was advertised via bulletin and online forums. The age of the chosen sample varied between 19 and 33 years, with a median of 21 years. For both AV and BL half of the participants were male, while TR was played by 14 females and six males. The median age for AV was 22 years and 21 years for the other two games. A similar age is important as stereoscopic vision abilities are strongly affected of aging [4]. All of the participants successfully passed a prior S3D vision test. The participants were offered free fruits, candy and beverages during the test sessions. They received "study points" they need to earn in order to conduct experiments in their courses. The evaluation was approved by our ethics board.

\section{Equipment}

The testing room was designed with a constantly low light source that did not interfere with the shutter glasses. The games were run on a quad-core desktop PC running Windows 7, with a GeForce GTX 275 graphics card and a Samsung 22" display at $120 \mathrm{~Hz}$ with 1680 x 1050 pixel resolution. Nvidia 3D Vision Driver version 6.14.12.5896 was used to generate $3 \mathrm{D}$ stereo. To later sort out any participants possibly faking play activities, the gaming sessions were recorded using Fraps (Version 3.0) by Beepa Pty Ltd. For the recordings, we used decreased resolutions and frame rate to ensure interactive frame rates $>25 \mathrm{fps}$. A MindSet headset was connected via bluetooth. We recorded the data using open source software Puzzlebox Synapse by Brainstorms. Due to stability problems, the software had to be controlled using an external PC. Thus, sound could not be provided using the Mindset and so participants had to wear additional in-ear headphones beneath the MindSet device. Since there was an impact on the subjective feel and comfort by wearing the full gear (glasses, headset, and earplugs, see Figure 1), the participants had to wear the 3D Vision glasses during both sessions (MV and SV). In monoscopic display, both shutters of the glasses are open, providing an image slightly darker than without glasses but brighter than the stereoscopic version and similar in color tone (altered towards yellow).

\section{Design and Procedure}

All participants had to play a game in both vision modes (SV, MV) in a within-subject design. The sequence of the vision modes was randomized to alleviate possible learning effects and a potential impact from small differences between first and second levels. The population was randomly broken into three groups of twenty users, where each group played one of the three games (AV, BL, TR) in both vision modes. The purpose of this decision was to enforce a comparison between vision modes, not between games. It further allowed us to reduce duration times of the testing sessions and to minimize impact from personal preference. The order of vision modes was randomized in a mixed design, where half of the participants started with SV and the other half with MV, equally distributed across games and gender. To minimize impact from personal preference for a certain game or genre, the games were randomly selected and equally distributed across gender. 


\section{Metrics}

Our choice of metrics was based on Affective Ludology [21], an approach which propagates a mixture of subjective self-reporting tools and objective psycho-physiological metrics to assess both cognitive and emotional sides of gameplay experience. In addition to demographic data, we asked participants about prior experience with gaming as well as with stereoscopic entertainment (games, TV, cinema). At the end of each trial, each participant was asked for their personal preference of vision modus and problems experienced during the sessions.

To assess user experience of game play, several tools have been proposed, mostly questionnaires (e.g. [2]) and/or psycho-physiological data [5]. We used the Game Experience Questionnaire's (GEQ) ${ }^{2}$ core questionnaire module. It contains seven dimensions: Immersion (Imm), Flow (Flo), Competence (Com), Tension (Ten), Challenge (Cha), Positive Affect (PoA), and Negative Affect (NeA). These are each measured using five to six items per dimension (36 in total), each on 1-to-5 disagree-agree response Likert-scales. We used the German version provided by Nacke [21]. The GEQ further includes a social presence module and a post-game module which were discarded in this study to shorten the overall test duration.

Presence was measured by the MEC Spatial Presence Questionnaire (MEC-SPQ) [31]. It includes four process factors: Attention Allocation (AA), Spatial Situation Model (SSM), Spatial Presence:Self Location (SP:SL) and Spatial Presence:Possible Actions (SP:PA). Two additional variables relating to states and actions (Higher Cognitive Involvement (HCI) and Suspension of Disbelief (SoD)) are also part of the MEC-SPQ. We used the short version offering four items for each scale.

Possible dizziness or other subjective disorders were measured using the Simulator Sickness Questionnaire (SSQ) [15]. It contains three dimensions, Nausea (Nau), Oculomotor (Ocu), and Disorientation (Diso), measured through 16 items on a 4-level Likert scale (ranging from "no symptoms" to "severe symptoms"). The three dimensions are then combined to create a Total SSQ metric (Tot). In our study, we used the German translation previously applied by Mehlitz [18].

Psychophysiological data was acquired using the NeuroSky MindSet. The headset measures electroencephalogram (EEG) data using contact dots. Three of these dots are included in the left ear cushion while the forth dot rests on the subject's forehead. The data consists of custom interpretations in so-called eSense meters: Attention (Att) and Meditation (Med). Although little data on the validity of these scales is available, previous work indicates positive

${ }^{2}$ GEQ, developed by K. Poels, W. A. IJsselsteijn, and Y. A. $\mathrm{W}$ de Kort at the Game Experience Lab Eindhoven (NL), (http://www.gamexplab.nl) in the European project FUGA. correlations of Att and Med with self-reported attention [25] and stress levels [3] .

\section{Procedure}

All participants were informed about the aim and procedure of the study and had to sign an agreement including a list of possibly occurring symptoms such as epilepsy, vision disorders or dizziness. All participants were tested for stereoscopic vision through the medical test image provided by the Nvidia driver settings. This introduction took about seven minutes on average. The experiment began with a five-minute introduction to the experiment and the signature of the participation agreement. In the following ten minutes, the participants completed a questionnaire at another PC that focused on demographic data and personal prior experience with games and stereoscopic vision. The participants were then seated in front of the gaming PC. They were introduced to the gear (3D vision glasses, MindSet and earbuds) and were asked to remain seated in a constant position. Before the tests started, the game was presented and could be tested for about ten minutes, until the participant understood the core game mechanics and was able to control and play for her- or himself.

Each of the two following gaming sessions, one per vision mode, lasted about fifteen minutes. During play, the participants were left alone by the experimenters, who could not see the current display content. Each gaming session was followed by a questionnaire session of about fifteen minutes. The whole experiment took about 1.5 hours per participant.

\section{RESULTS AND ANALYSIS}

\section{Demographic information}

We collected data of frequency of play for all participants. Ten participants never played computer games $(16.7 \%), 32$ played 1-5 hours per week (53.3\%), 11 played 6-10 hours per week (18.3\%), and seven played 11-20 hours per week $(11.7 \%)$. None of our participants played more than 20-30 or more than 30 hours per week. The typical weekly playing duration was 4.9 hours across all participants. Male subjects (7.3 hours per week) played noticeably more frequently than females ( 3 hours per week).

The participants had the most genre-related experience with Jump'n'Run (38\%), Strategy (37\%), RPG (33\%), and Puzzles (32\%). More than a fifth listed Action RPG (23\%), Ego-Shooter (23\%), and Adventures (20\%), followed by Sports (18\%), Simulations (17\%), Racing (13\%), Fighting (11\%), and Education games (2\%). Again, experience with genre differed between both genders: females are better used to Puzzle games (38\%), Jump'n'Run (32\%), and Strategy/RPG games (21\% each). Males had generally higher experience levels, maximizing in Strategy (58\%), Ego-Shooter/RPG (50\% each), and Jump'n'Run/ActionShooter ( $46 \%$ each). Considering BL, Racing was better known among males (27\%) than among females (3\%). 


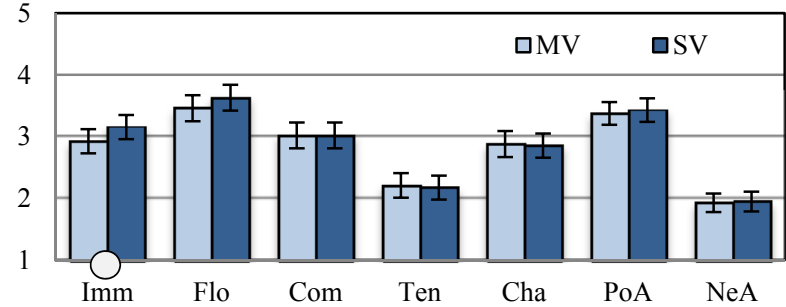

Figure 2. GEQ data across all groups- a significant difference between mono and stereo vision was only found in Immersion. (Error bars show 95\% CI, $\bigcirc_{=p<.05 ;} \bigcirc_{=p<.01 ;}=p<.001$ )

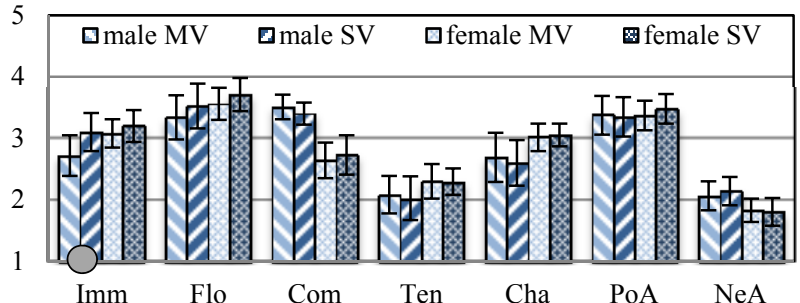

Figure 3. GEQ Data - gender vs. vision mode. No effects on female subjects. Males experienced higher immersion.

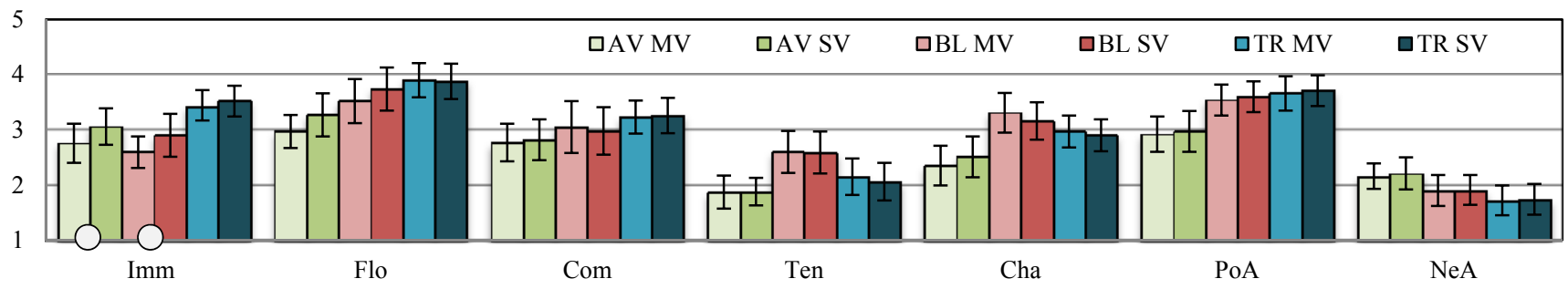

Figure 4. GEQ data - game vs. vision mode: Immersion in Avatar and Blur shows significant differences between vision modes.

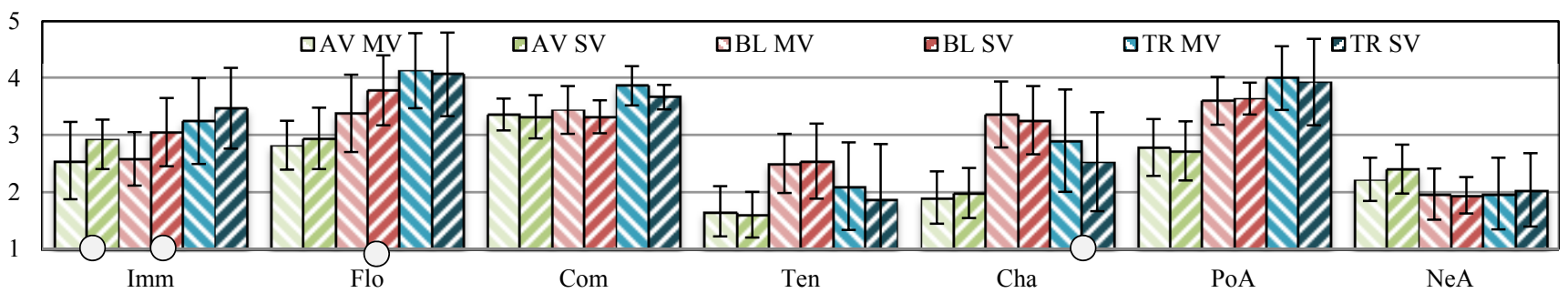

Figure 5. GEQ data - male subjects only - game vs. vision mode: stereoscopy impacts male subjects' Immersion in Avatar, Immersion and Flow in Blur, and Challenge in Trine.

Only a few of the participants had played the games we tested. Seven reported to have previously played AV one to ten times $(11.7 \%)$, one had played BL one to ten times $(1.7 \%)$ and two had played it for more than 25 times $(3.3 \%$, they were in the AV group). One player had previously played TR one to ten times (1.7\%).

Prior experience with S3D was collected for cinema, TV and digital games. Of the 60 participants, four had never seen a 3D cinema movie, 34 one to three times and 22 four to nine times. Fifteen had previously watched 3DTV one to three times, one had watched four to nine times, the others never. Only six participants had previously played S3D games $(10 \%)$. These results were similar for both genders.

\section{GEQ}

Comparing SV with MV, the GEQ data revealed higher mean values of Immersion, Flow, Positive Affect, and Negative Affect and lower mean values in Tension and Challenge in the stereo condition (see Figure 2). A dependent two-sample T-Test revealed significant differences only for Immersion $\left(\mathrm{t}_{59}=-3.293, \mathrm{p}<.01\right)$.

Looking at these results for each gender (see Figure 3), a dependent two-sample T-Test confirmed the significant difference only among male participants $\left(\mathrm{t}_{25}=-3.676, \mathrm{p}<.01\right)$ showing a higher immersion in SV than in MV. Female participants showed no significant difference.

We found differences for the three particular games (see Figure 4), as only $\mathrm{AV}$ and $\mathrm{BL}$ revealed a significantly higher Immersion (AV: $\mathrm{t}_{19}=-2.348, \mathrm{p}<.05 ; \mathrm{BL}: \mathrm{t}_{19}=-2.291$, $\mathrm{p}<.05)$. More effects became visible for the male group only (see Figure 5). Again, Immersion in SV was higher than in MV for AV $\left(\mathrm{t}_{9}=-2.318, \mathrm{p}<.05\right)$ and $\mathrm{BL}\left(\mathrm{t}_{9}=-2.318\right.$, $\mathrm{p}<.05$ ). For males playing BL, a Wilcoxon-Signed-Rank test showed a significantly higher Flow value in SV than in MV $(Z=2.214, p<.05)$. In $T R$, male subjects experienced Challenge significantly lower under SV than under MV condition $\left(\mathrm{t}_{5}=3.841, \mathrm{p}<.05\right)$. No effects were found in the female group.

\section{MEC-SPQ}

All presence dimensions of the MEC-SPQ with one exception had higher mean values in SV than in MV (see Figure 6). Significant differences were found in AA $(Z=2.522, p<.05)$, SP:SL $(Z=5.071, p<.001)$, and SP:PA $\left(\mathrm{t}_{59}=-4.707, \mathrm{p}<.001\right)$. The differences in SSM and SoD were not significant. In contrast, the mean values of HCI in stereo were significantly below those in the mono condition $(Z=-4.568, \mathrm{p}<.001)$. 


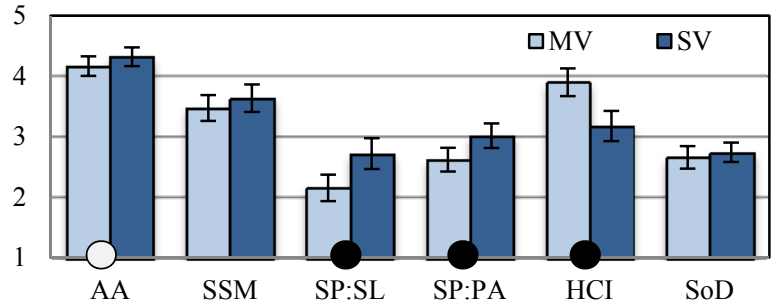

Figure 6. MEC-SPQ with Attention Allocation (AA), Spatial Situation Model (SSM), Spatial Presence:Self Location (SP:SL), Spatial Presence:Possible Actions(SP:PA), Higher Cognitive Involvement (HCI), Suspension of Disbelief (SoD).

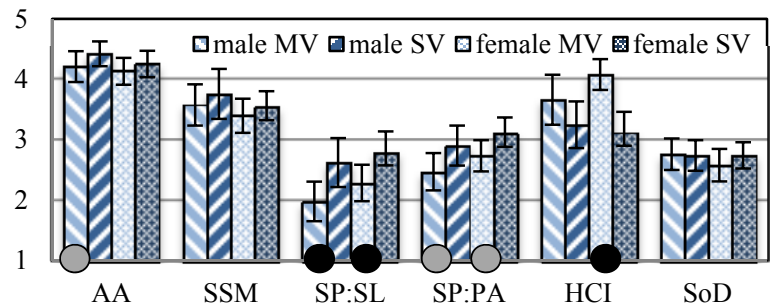

Figure 7. MEC-SPQ vision mode $x$ gender. Different characteristics in presence for male and female subjects. (Error bars show 95\% CI, $\bigcirc_{=\mathbf{p}<.05 ;} \bigcirc_{=\mathbf{p}<.01 ;}=\mathbf{p}<.001$ )

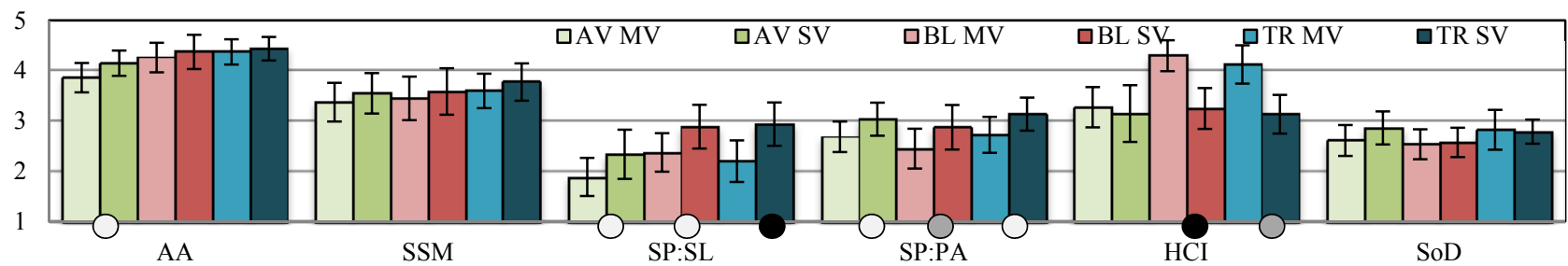

Figure 8. MEC-SPQ games $x$ vision mode. All games provide enhanced presence in SV with some differences in AA and HCI.

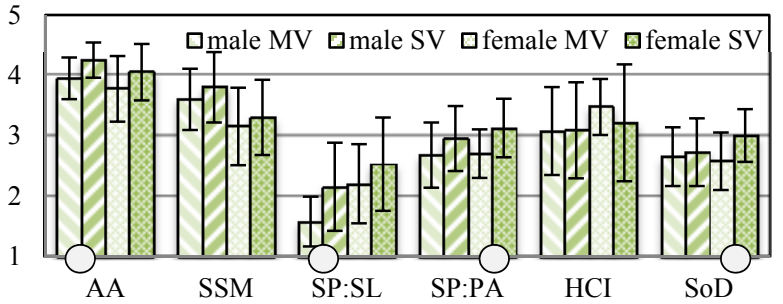

Figure 9. MEC-SPQ Avatar gender vs. vision mode. Both gender groups were affected by $\mathrm{SV}$.

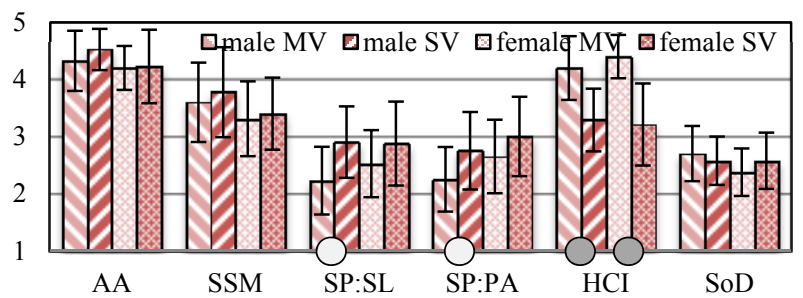

Figure 10. MEC-SPQ Blur gender vs. vision mode. More effects could be found in the male group.

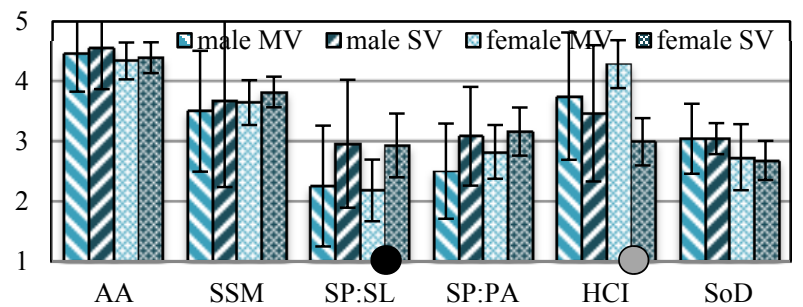

Figure 11. MEC-SPQ Trine gender vs. vision mode. Only female users report differences between $\mathrm{MV}$ and $\mathrm{SV}$.
As with the GEQ, we extracted gender specific differences (see Figure 7): Female subjects had significantly higher values in SV than in MV for SP:SL $\left(\mathrm{t}_{33}=-4.361, \mathrm{p}<.001\right)$ and SP:PA $\left(\mathrm{t}_{33}=-3.428, \mathrm{p}<.01\right)$. They had significantly lower values of HCI in stereo than in mono $(Z=-4.095$, $\mathrm{p}<.001)$. Male subjects had significantly higher values in stereo for AA $(Z=2.742, p<.01)$, SP:SL $(Z=3.654, p<.001)$, and SP:PA $\left(\mathrm{t}_{25}=-3.175, \mathrm{p}<.01\right)$.

Looking at the particular games (see Figure 8), SV increased presence in all three games: We found significantly higher values of Spatial Presence:Self Location (AV: $\mathrm{Z}=2.459, \mathrm{p}<.05$, BL: $\mathrm{t}_{19}=-2.848, \mathrm{p}<.05$, TR: $\left.\mathrm{t}_{19}=-5.902, \mathrm{p}<.001\right)$ and Spatial Presence:Possible Action $\left(\mathrm{AV}: \mathrm{t}_{19}=-2.483, \mathrm{p}<.05, \mathrm{BL}: \mathrm{t}_{19}=-3.023, \mathrm{p}<0.01, \mathrm{TR}: \mathrm{t}_{19}=-\right.$ $2.554, \mathrm{p}<.05)$ in stereo than in mono. Again, the games evoked different characteristics as well. Only AV induced significantly higher values of Attention Allocation in SV than in MV $\left(\mathrm{t}_{19}=-2.596, \mathrm{p}<.05\right)$. Only BL and TR showed significantly lower values of Higher Cognitive Involvement in $S V$ than in MV (BL: $Z=-3.733, p<.001$, TR: $Z=-3.136$, $\mathrm{p}<.01$ ). Again, means of SSM and SoD were not significantly different within any of the game groups.

Interestingly, these effects showed different characteristics when analyzed for each gender. AV (see Figure 9) affected both genders, but in different aspects: stereo significantly pushed SP:PA $\left(\mathrm{t}_{9}=-2.547, \mathrm{p}<.05\right)$ and $\operatorname{SoD}(\mathrm{Z}=2.214, \mathrm{p}<.05)$ for female users, while it increased AA $\left(\mathrm{t}_{9}=-2.714, \mathrm{p}<.05\right)$ and SP:SL $(Z=1.973, p<.05)$ for male users. In BL (see Figure 10), stereo had more effect for the male group, where SP:SL $\left(\mathrm{t}_{9}=-2.976, \mathrm{p}<.05\right)$ and SP:PA $\left(\mathrm{t}_{9}=-2.535\right.$, $\mathrm{p}<.05)$ were significantly higher in SV than in MV. HCI was significantly lower for both male $\left(\mathrm{t}_{9}=3.636, \mathrm{p}<.01\right)$ and 
female subjects $\left(t_{9}=3.952, p<.01\right)$. In contrast to the TR group (see Figure 11), stereo only affected female subjects whose SP:SL $\left(\mathrm{t}_{9}=-6.101, \mathrm{p}<.001\right)$ was significantly higher and $\mathrm{HCI}(\mathrm{Z}=-3.171, \mathrm{p}<.01)$ was lower compared to MV.

\section{SSQ}

Stereoscopic vision also had an impact on the amount of experienced symptoms of simulator sickness (see Figure 12): The mean value of Total SSQ was significantly higher $(\mathrm{Z}=2.353, \mathrm{p}<.05)$ in $\mathrm{SV}$ than in MV. All three subscales (Nausea, Oculomotor and Disorientation) also showed higher SV mean values, but only the difference in Ocu was significant $(\mathrm{Z}=2.853, \mathrm{p}<.01)$. Surprisingly, this increase in symptoms was only reported by the male subjects. They showed significantly higher values in SV for all subscales and the total value (Nau: $Z=2.138, p<.05$; Ocu: $Z=3.154$, $\mathrm{p}<.01$; Diso: $Z=2.205, \mathrm{p}<.05$; Tot: $Z=3.241, \mathrm{p}<.01$ ). The female subjects reported lower mean values in SV than in $\mathrm{MV}$, but these differences were not significant.

Within the game conditions over all participants, no significant differences between SV and MV were found. Only when focusing on the male group, we found SV causing significantly more symptoms in total for AV $(\mathrm{Z}=2.325, \mathrm{p}=.05)$ and $\mathrm{BL}\left(\mathrm{t}_{9}=-3.503, \mathrm{p}<.01\right)$. For male subjects playing $\mathrm{AV}$, Ocu $(\mathrm{Z}=2.111, \mathrm{p}<.05)$ and Diso $(\mathrm{Z}=2.032, \mathrm{p}<.05)$ were higher in SV than in MV. For males playing BL, Nau $(Z=2.121, \mathrm{p}<.05)$, Ocu $(\mathrm{t} 9=-2.954, \mathrm{p}<.05)$, and Diso $(Z=2.271, p<.05)$ were significantly higher in $S V$. No effects were found for Trine, nor for the female group playing any game.

\section{MindSet}

We had to exclude eight of the subjects due to erroneous data in at least one condition. Of the remaining sample, 29 were female and 23 were male. We interpreted the data using arithmetic mean values for Attention and Meditation of each session. Attention showed a significantly lower value in $\mathrm{SV}$ than in MV $\left(\mathrm{t}_{51}=4.338, \mathrm{p}<.01\right)$. This effect (see Figure 13) can be found in both gender groups (female: $\mathrm{t}_{28}=3.450, \mathrm{p}<.01 ;$ male: $\mathrm{t}_{22}=2.646, \mathrm{p}<.05$ ). Regarding the games (see Figure 14), Attention was significantly lower while playing $\mathrm{AV}(\mathrm{Z}=-2.166, \mathrm{p}<.05)$ and $\mathrm{BL}\left(\mathrm{t}_{18}=4.307\right.$, $\mathrm{p}<.001)$ in SV than playing in MV. TR did not evoke any significant differences.

\section{S3D Preference and Other Findings}

Comparable to previous findings, 51 participants (85\%) had more fun with the SV mode. Of the nine others, five had played BL, three had played AV and one TR (6 male, 3 female). We also asked for any problems during play. Our subjects reported more problems with game controls $(36.7 \%)$ and the gear $(33.3 \%)$ than with the display (16.7\%). For some participants (11.7\%), the game difficulty was too low. We further collected both positive and negative comments (see Table 1). The positive ones state a new and fun experience with a more spatial connotation; the negative remarks were related to comfort and reported unsuitable game interaction for stereoscopic vision (quick camera movements, distracting HUD display).

\begin{tabular}{ll}
\hline Positive comments & Negative comments \\
\hline - more fun & - more eye strain \\
- more intensive game feel & - uncomfortable glasses \\
- better spatial visualization & - 3D effects distract from game \\
- more spatial feeling & play \\
- more plasticity of the & - 3D display is unsuitable for \\
$\quad$ environment & quick camera movements, only \\
- more immersive impression & great when you stand still \\
- new quality of play experience & - looking at HUD information is \\
- much more realistic & easier in Mono 3D \\
\hline
\end{tabular}

Table 1. Positive and negative comments on S3D gaming.

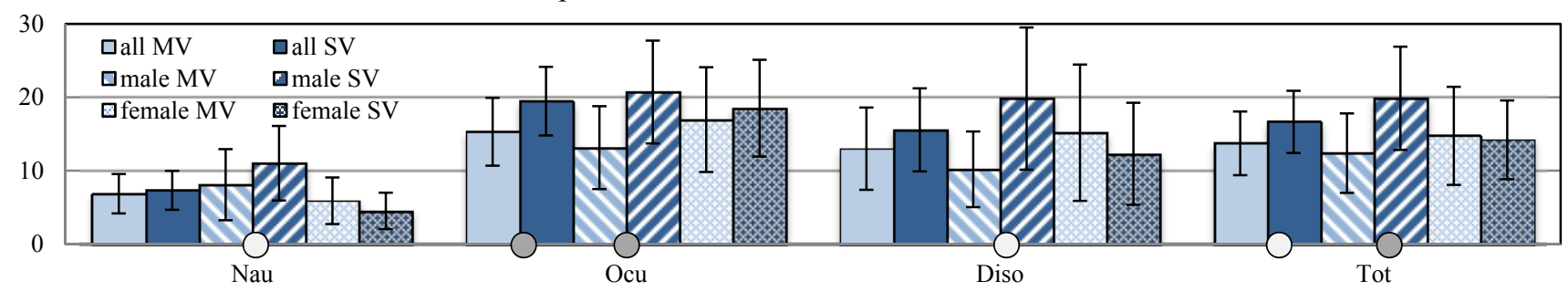

Figure 12. SSQ - all groups and gender vs. vision mode. SV had effect on all participants, but more significantly on male subjects. (Error bars show 95\% CI, $\bigcirc_{=\mathbf{p}<.05 ;} \mathrm{O}_{\mathbf{p}}<.01 ; \mathrm{O}_{\mathbf{p}}<.001$ )

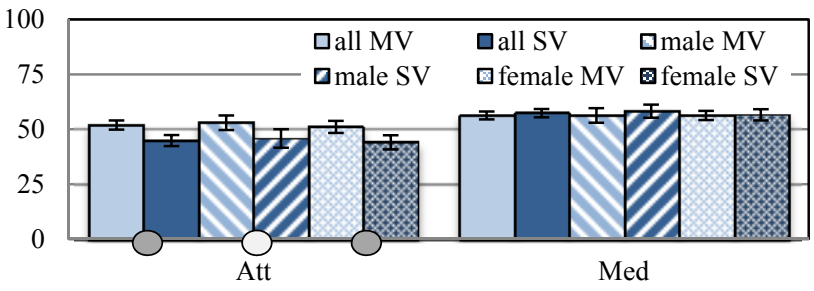

Figure 13. MindSet - gender vs. vision mode. Attention significantly decreases among all participants in SV.

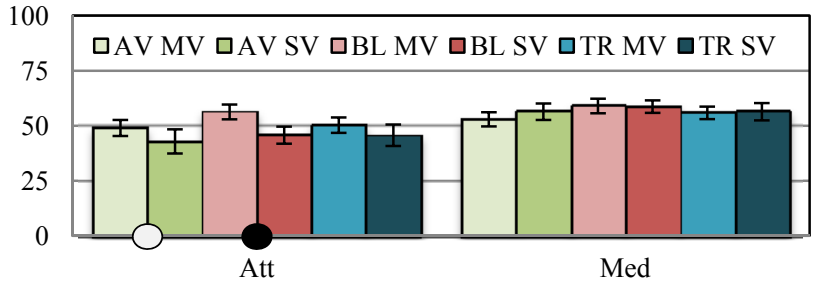

Figure 14. MindSet - games vs. vision mode. The effects for Attention through SV were only confirmed in AV and BL. 


\begin{tabular}{|c|c|c|c|c|c|c|c|c|}
\hline & \multicolumn{2}{|c|}{ Total } & \multicolumn{2}{|c|}{ Avatar } & \multicolumn{2}{|c|}{ Blur } & \multicolumn{2}{|c|}{ Trine } \\
\hline & male & female & male & female & male & female & male & female \\
\hline \multirow{2}{*}{ GEQ } & \multicolumn{2}{|c|}{$\mathrm{Imm}+$} & \multicolumn{2}{|c|}{$\mathrm{Imm}+$} & \multicolumn{2}{|c|}{$\mathrm{Imm}+$} & \multirow{2}{*}{\multicolumn{2}{|c|}{ Cha- }} \\
\hline & $\mathrm{Imm}+$ & & $\mathrm{Imm}+$ & & $\mathrm{Imm}+, \mathrm{Flo}+$ & & & \\
\hline \multirow[b]{2}{*}{$\begin{array}{l}\text { MEC- } \\
\text { SPQ }\end{array}$} & \multicolumn{2}{|c|}{$\mathrm{AA}^{+}, \mathrm{SP}: \mathrm{SL}+, \mathrm{SP}: \mathrm{PA}+, \mathrm{HCI}-$} & \multicolumn{2}{|c|}{$\mathrm{AA}+, \mathrm{SP}: \mathrm{SL}+, \mathrm{SP}: \mathrm{PA}+$} & \multicolumn{2}{|c|}{ SP:SL+, SP:PA+, HCI- } & \multicolumn{2}{|c|}{ SP:SL+, SP:PA+, HCI- } \\
\hline & $\begin{array}{l}\text { AA+, } \\
\text { SP:SL+, } \\
\text { SP:PA+ }\end{array}$ & $\begin{array}{c}\text { SP:SL+, } \\
\text { SP:PA+, } \\
\text { HCI- }\end{array}$ & $\mathrm{AA}+, \mathrm{SP}: \mathrm{SL}+$ & $\begin{array}{c}\text { SP:PA+, } \\
\text { SoD+ }\end{array}$ & $\begin{array}{l}\text { SP:SL+, } \\
\text { SP:PA+, } \\
\text { HCI- }\end{array}$ & HCI- & & $\begin{array}{l}\text { SP:SL+, } \\
\text { HCI- }\end{array}$ \\
\hline \multirow[b]{2}{*}{ SSQ } & \multicolumn{2}{|c|}{ Tot,+ Ocu +} & & & & & & \\
\hline & $\begin{array}{c}\mathrm{Nau}^{+}, \mathrm{Ocu}^{+}, \\
\mathrm{Diso}^{+}, \mathrm{Tot}^{+}\end{array}$ & & $\begin{array}{c}\mathrm{Ocu}^{+}, \mathrm{Diso}^{+}, \\
\text {Tot+ }+\end{array}$ & & $\begin{array}{l}\mathrm{Nau}^{+}, \mathrm{Ocu}+, \\
\text { Diso+, Tot+ }\end{array}$ & & & \\
\hline \multirow{2}{*}{ MindSet } & \multicolumn{2}{|c|}{ Att- } & \multirow{2}{*}{\multicolumn{2}{|c|}{ Att- }} & \multirow{2}{*}{\multicolumn{2}{|c|}{ Att- }} & & \\
\hline & Att- & Att- & & & & & & \\
\hline
\end{tabular}

Table 2: The impact of stereoscopic vision on player experience for all metrics across the particular groups (games and gender) $(+$ means a significant increase, - indicates a significant decrease compared to monoscopic presentation).

\section{DISCUSSION AND FUTURE WORK}

According to the GEQ data alone, stereoscopy resulted in increased Immersion and thus enhanced game experience, confirming our first hypothesis. Most of the other results in MEC-SPQ and SSQ correspond to previous findings on stereoscopic applications (i.e., stereoscopic games) seem to induce an increased spatial presence, but cause a higher level of simulator sickness than monoscopic games. This also confirms our second hypothesis. Interestingly, and other than what we had expected based on previous findings $[16,24]$, all of these effects are strongly altered by the choice of games which clearly rejects our third hypothesis. In addition we found a strong impact from the gender of the players, which we did not expect to happen.

\section{The Impact of Game and Gender}

Stereoscopic vision affects particular users differently for varying games (as summarized in Table 2). GEQ only showed differences for male users: BL caused males to report higher Flow; in TR they experienced less Challenge when playing in S3D. SV seems to have more impact on Immersion in those games which offer a lot of depth animation (AV, BL) than in a side-scrolling game (TR). For the former two games, males were more strongly affected in game experience and simulator sickness than females.

Similarly, the MEC-SPQ had different impact for the games and gender. SV in BL increased presence primarily in males; SV in AV had an effect on presence in both gender groups. SV in TR had more impact in presence on females.

Effects of simulator sickness were found to be increased in $\mathrm{SV}$ for the whole sample, but again differently for the separate games and gender (i.e., males mainly playing AV and BL were affected). The TR group did not report any such impairment, nor did the female subjects which partly contradicts previous findings [12]. This effect could relate to the used display technology, as a previous study using a lenticular display did not find an impact on SSQ [24].

We consider the strong impact of SV across all metrics on males to be affected for two reasons. First, our male users stated a greater prior experience with game genres and report more frequent use of digital games than female subjects. Thus, the effect of SV could be also related to experience than just gender. A second aspect is a possible higher affinity of males for 3D animations and spatially complex environments and the known gender difference in spatial abilities (i.e., males can solve spatial problems faster and more accurately than females [6]).

At the same time however, SV was strongly preferred for any choice of game in both gender groups. Hence, it will require further research to allow forecasting preference and quality assessment from analyzing user experience characteristics. Our data does not indicate whether the distinctive preferences found may result from a possible novelty factor of stereoscopic games and may vanish with longer playing times.

\section{Attention in S3D Gaming}

An increased spatial presence was generally experienced while playing stereoscopic games. Players felt more directly located within the stereoscopic game and increasingly urged to actively participate. More attention was also allocated by the game. At the same time, higher cognitive involvement was reduced under SV, so players seem to less actively reflect their playing on a meta-level. As with game experience, these effects differed for the three games. While BL had more impact on male users, TR only affected female users in their spatial presence. In $\mathrm{AV}$, we found similar effects for both male and female users.

The MindSet data showed a lower mean Attention during SV game play. It is questionable how this decrease might be related with the MEC-SPQ values of increased AA and decreased Higher Cognitive Involvement in SV. We did not find any correlation of Attention with any other metric. However, as the MindSet is primarily designed as an input device in order to allow players active control of a computer using brain activities, we would rather expect this value to reflect self-induced or conscious attention. As such, it does not contradict the increase of AA found in the stereo MEC-SPQ which reports how a game allocates or draws attention of a player. Hence, we argue that stereoscopic presentation draws more attention but leads to less thoughtfulness and attentiveness, possibly contributing to a more intuitive and natural interaction with a game. A possible reason could be the generally more natural visual experience with stereoscopic images, also reported in [27]. 


\section{The Difference of S3D in Current Games and Movies}

Besides the Immersion effect, SV had only little overall impact on game experience as no other differences were found across all participants. In general, the GEQ is able to show such differences, visible when analyzing the variances between the three games: All but one GEQ-dimension were significantly different between the three games in $\mathrm{MV}$, correctly reflecting their differences. In SV however, only four of seven dimensions were different for the three games, they were effectively more similar to each other in $\mathrm{SV}$ than in MV in terms of game experience. We have to note that none of the evaluated games were primarily designed for stereoscopic vision. As previously stated, all current games have $\mathrm{SV}$ as an option and must remain playable without it [26].

In comparison, stereoscopic movies are thoroughly produced for S3D presentation, taking into account actual movie theater configurations and display settings [19]. They differ in parts (e.g., cutting, scene transitions) from their monoscopic versions. Movie makers can build on a long history of stereoscopic cinematography that directs visual attention towards certain depths of interest within a scene. These solutions are hardly applicable in games, where the camera and scene depths often change dynamically and interactively. Many current games further suffer from certain S3D rendering problems and interface artifacts.

\section{Goals and Actionable Steps for the Future}

Our goal in subsequent work will be to develop a game design which is experienced differently when presented in stereoscopic vision. The effects found here give a first direction, indicating an impact of depth animation, showing that SV can contribute to a particular game experience, when taking into account how the design affects the core target group. Future assessment should also examine if the given results for these games are valid for similar games, allowing for systematically estimating a benefit of stereoscopy for a certain type of game or genre.

\section{CONCLUSIONS}

This paper provides a comprehensive study on the impact of stereoscopy on gaming experience. We analyzed three different games by comparing two vision modes using GEQ, MEC-SPQ, SSQ and EEG-related metrics. Our results contain three major contributions. First, we confirm previous findings that playing games in $\mathrm{S} 3 \mathrm{D}$ is clearly preferred over mono. It increases experiences of presence and immersion while causing more symptoms of simulator sickness. Second, we show for the first time that these effects depend strongly on the game and the gender of the user. For a racing game with compelling 3D camera movement, we found more impact on male users, while a side-scrolling-game with less spatial animations had more effect on female users. Avatar, offering dynamic 3D scenes with side- and depth-relative camera movements, affected both genders. Third, a higher Attention Allocation in S3D was accompanied by a reduced Higher Cognitive Involvement, indicating a more direct and unconscious interaction with stereoscopic games, effectively offering a more natural player experience. Future research needs to confirm these results for other games, helping to understand which particular design characteristics actually contribute to a more compelling experience through stereoscopic vision.

\section{ACKNOWLEDGEMENTS}

Many thanks go to our students, who helped with the experiments, especially Liane Bölicke, Christian Hierhammer and Sebastian Kirch, and to the participants.

\section{REFERENCES}

1. Bernhaupt, R. User Experience Evaluation in Entertainment. In R. Bernhaupt, ed., Evaluating User Experience in Games Concepts and Methods. Springer London, 2010, 3-7.

2. Brockmyer, J.H., Fox, C.M., Curtiss, K.A., McBroom, E., Burkhart, K.M., and Pidruzny, J.N. The development of the Game Engagement Questionnaire: A measure of engagement in video game-playing. Journal of Experimental Social Psychology, 45 (2009), 624-634.

3. Crowley, K., Sliney, A., Pitt, I., and Murphy, D. Evaluating a Brain-Computer Interface to Categorise Human Emotional Response. 2010 10th IEEE International Conference on Advanced Learning Technologies, (2010), 276-278.

4. Dodgson, N. Variation and extrema of human interpupillary distance. Stereoscopic Displays and Virtual Reality Systems XI 5291, (2004), 36-46.

5. Drachen, A., Nacke, L.E., Yannakakis, G., and Pedersen, A.L. Correlation between Heart Rate, Electrodermal Activity, and Player Experience in FirstPerson Shooter games. Proceedings of the 5th ACM SIGGRAPH Symposium on Video Games, ACM New York, NY, USA (2010), 49-54.

6. Gaulin, S.J.C. and McBurney, D.H. Evolutionary Psychology. Prentice Hall, 2003.

7. Hoffman, D., Girshick, A., Akeley, K., and Banks, M.S. Vergence-accommodation conflicts hinder visual performance and cause visual fatigue. Journal of Vision 8,3 (2008), 1-30.

8. Holliman, N., Froner, B., and Liversedge, S. An application driven comparison of depth perception on desktop 3D displays. Proceedings of SPIE, January (2007), 64900H-64900H-12.

9. Howarth, P. Potential hazards of viewing 3-D stereoscopic television, cinema and computer games: a review. Ophthalmic \& physiological optics : the journal of the British College of Ophthalmic Opticians (Optometrists) 31, 2 (2011), 111-22.

10. Hubona, G.S., Wheeler, P.N., Shirah, G.W., and Brandt, $\mathrm{M}$. The relative contributions of stereo, lighting, and background scenes in promoting 3D depth visualization. 
ACM Transactions on Computer-Human Interaction 6, 3 (1999), 214-242.

11. Häkkinen, J., Kawai, T., Takatalo, J., Leisti, T., Radun, J., and Hirsaho, A. Measuring Stereoscopic Image Quality Experience with Interpretation Based Quality Methodology. System 6808, January (2008), 27-31.

12. Häkkinen, J., Pölönen, M., Takatalo, J., and Nyman, G. Simulator sickness in virtual display gaming.

Proceedings of the 8th conference on Human-computer interaction with mobile devices and services MobileHCI '06, ACM Press (2006), 227-230.

13. IJsselsteijn, W., Ridder, H. de, Freeman, J., Avons, S.E.E., and Bouwhuis, D. Effects of Stereoscopic Presentation, Image Motion, and Screen Size on Subjective and Objective Corroborative Measures of Presence. Presence: Teleoperators \& Virtual Environments 10, 3 (2001), 298-311.

14. Jumisko-Pyykko, S., Utriainen, T., Strohmeier, D., Boev, A., and Kunze, K. Simulator sickness - Five experiments using autostereoscopic mid-sized or small mobile screens. 2010 3DTV-Conference: The True Vision - Capture, Transmission and Display of $3 D$ Video, IEEE (2010), 1-4.

15. Kennedy, R., Lane, N., and Berbaum, K. Simulator Sickness Questionnaire: An enhanced method for quantifying simulator sickness. The International Journal of Aviation Psychology 3, 3 (1993), 203-220.

16. LaViola Jr, J.J. and Litwiller, T. Evaluating the benefits of $3 \mathrm{~d}$ stereo in modern video games. Proceedings of the 2011 annual conference on Human factors in computing systems, ACM (2011), 2345-2354.

17. Lambooij, M.T.M., IJsselsteijn, W. a., and Heynderickx, I. Visual Discomfort in Stereoscopic Displays: A Review. Proceedings of SPIE 6490, May 2010 (2007), 64900I-64900I-13.

18. Mehlitz, M.A. Aufbau eines medizinischen VirtualReality-Labors und Entwicklung eines VR-gestützten neuropsychologischen Testsystems mit einer präklinischen und klinischen Evaluationsstudie. 2004. http://webdoc.sub.gwdg.de/diss/2004/mehlitz/mehlitz.p df.

19. Mendiburu, B. 3D movie making: stereoscopic digital cinema from script to screen. Elsevier, 2009.

20. Nacke, L.E. and Drachen, A. Towards a Framework of Player Experience Research. Proceedings of EPEX'11 at FDG 2011, Bordeux, France, (2011).

21. Nacke, L.E. Affective Ludology. Technology, (2010).

22. Obrist, M., Wurhofer, D., Förster, F., et al. Perceived 3DTV Viewing in the Public: Insights from a ThreeDay Field Evaluation Study. Human Factors, (2011), 167-176.
23. Palmisano, S. Consistent stereoscopic information increases the perceived speed of vection in depth. Perception 31, 4 (2002), 463-80.

24. Rajae-Joordens, R.J.E. Measuring experiences in gaming and TV applications. Probing Experience, (2008), 77-90.

25. Rebolledo-Mendez, G., Dunwell, I., Martinez-Mirón, E., et al. Assessing NeuroSky's Usability to Detect Attention Levels in an Assessment Exercise. Lecture Notes in Computer Science 5610, Human-Computer Interaction. New Trends (2009), 149-158.

26. Schild, J. and Masuch, M. Fundamentals of Stereoscopic 3D Game Design. Proceedings of ICEC 2011, Ifip (2011), 155-160.

27. Seuntiens, P.J., Heynderickx, I., IJsselsteijn, W.A., et al. Viewing experience and naturalness of 3D images. Proceedings of SPIE 6016, 0 (2005), 601605-601605-7.

28. Shibata, T., Kim, J., Hoffman, D.M., and Banks, M.S. The zone of comfort: Predicting visual discomfort with stereo displays. Journal of vision 11, 8 (2011), 1-29.

29. Takatalo, J., Kawai, T., Kaistinen, J., Nyman, G., and Häkkinen, J. User Experience in 3D Stereoscopic Games. Media Psychology 14, 4 (2011), 387-414.

30. Tam, W.J., Stelmach, L.B., and Corriveau, P.J. Psychovisual aspects of viewing stereoscopic video sequences. Proceedings of SPIE 3295, SPIE (1998), 226-235.

31. Vorderer, P., Wirth, W., Gouveia, F.R., et al. MEC spatial presence questionnaire (MEC-SPQ): Short documentation and instructions for application. Report to the European Community, Project Presence: MEC (IST-2001-37661), (2004).

32. Ware, C. and Mitchell, P. Reevaluating stereo and motion cues for visualizing graphs in three dimensions. Proceedings of the 2nd symposium on Applied perception in graphics and visualization - APGV '05, ACM Press (2005), 51.

33. Woods, A., Docherty, T., and Koch, R. Image distortions in stereoscopic video systems. Stereoscopic displays and applications IV 1915, February 1993 (1993), 36-48.

34. You, J., Jiang, G., Xing, L., and Perkis, A. Quality of Visual Experience for 3D Presentation - Stereoscopic Image. In M. Mrak, M. Grgic and M. Kunt, eds., HighQuality Visual Experience. Springer Berlin Heidelberg, Berlin, Heidelberg, 2010, 51-77.

35. Zachara, M. and Zagal, J.P. Challenges for Success in Stereo Gaming: A Virtual Boy Case Study. Proceedings of the International Conference on Advances in Computer Entertainment Technology, ACM (2009), 99-106. 